\title{
Memristive behavior in one-dimensional hexagonal boron nitride/carbon nanotube heterostructure assemblies
}

Hiroo Suzuki*广, Misaki Kishibuchi ${ }^{\dagger}$, Kazuma Shimogami, Mitsuaki Maetani, Kyohei Nasu, Tomohiro Nakagawa, Yuichiro Tanaka, Hirotaka Inoue and Yasuhiko Hayashi

Graduate school of Natural Science and Technology, Okayama University, 3-1-1

Tsushima-naka, Kita-ku, Okayama 700-8530, Japan

*Email: hiroo.suzuki@okayama-u.ac.jp 


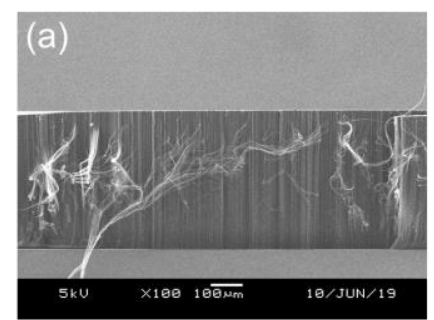

(e)

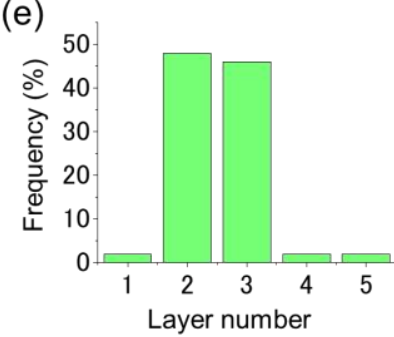

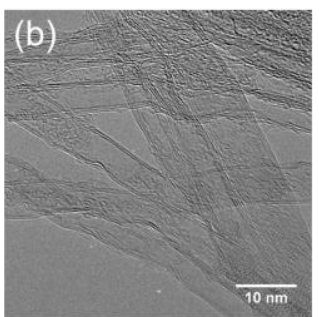

(f) 25

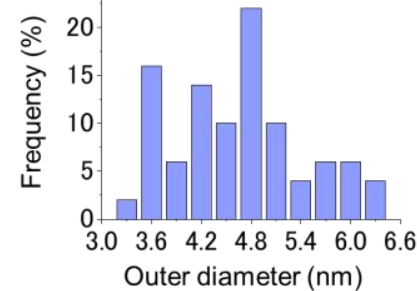

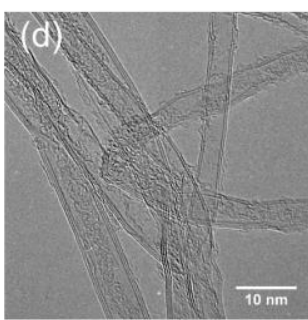

(g) 25

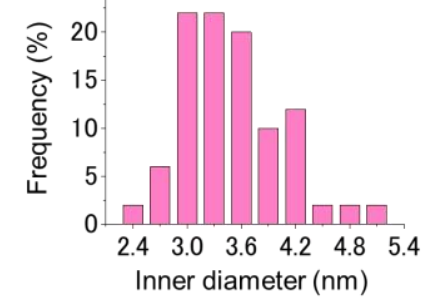

Figure S1. Structure of pristine CNTs. (a) Cross-section SEM image of the CNT forest. (b)-(d) TEM images of pristine CNTs. (e-g) Frequency of (e)layer number, (f)outer diameter, (g)inner diameter of pristine CNTs.

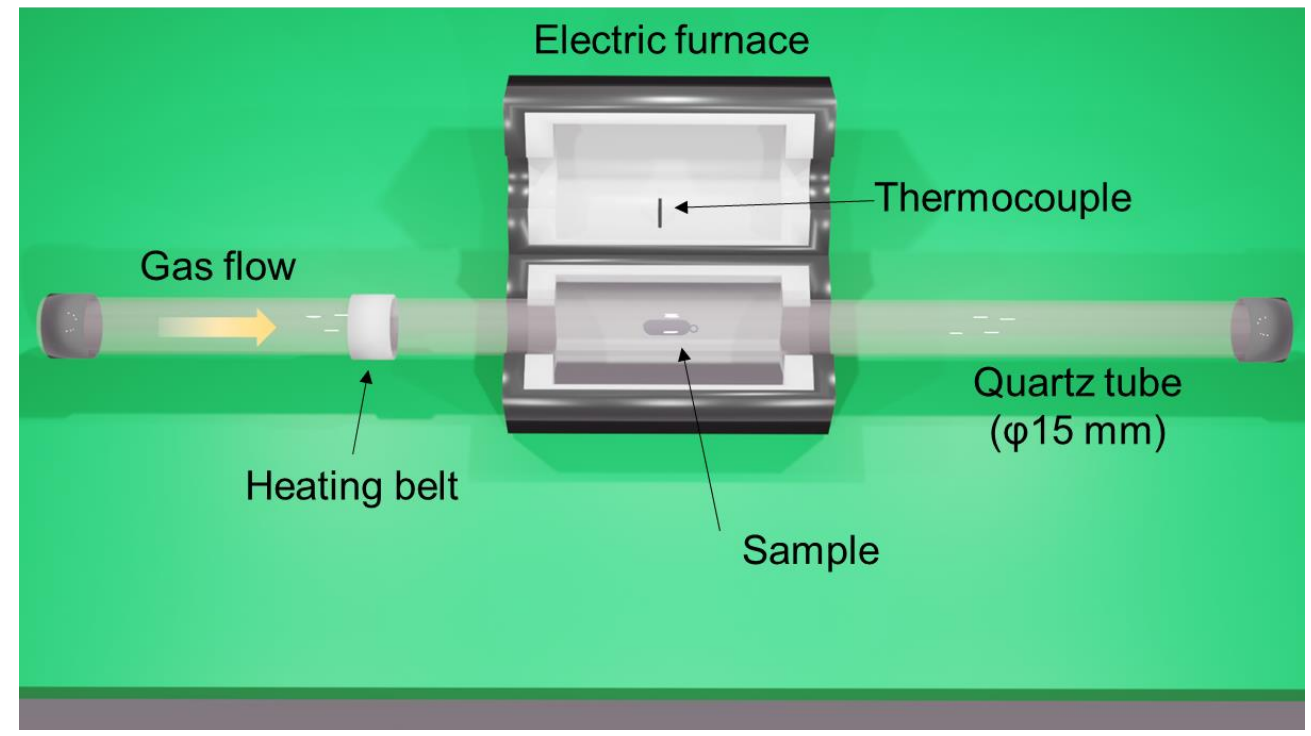

Figure S2. Schematic image of powder CVD device for hBN growth. 

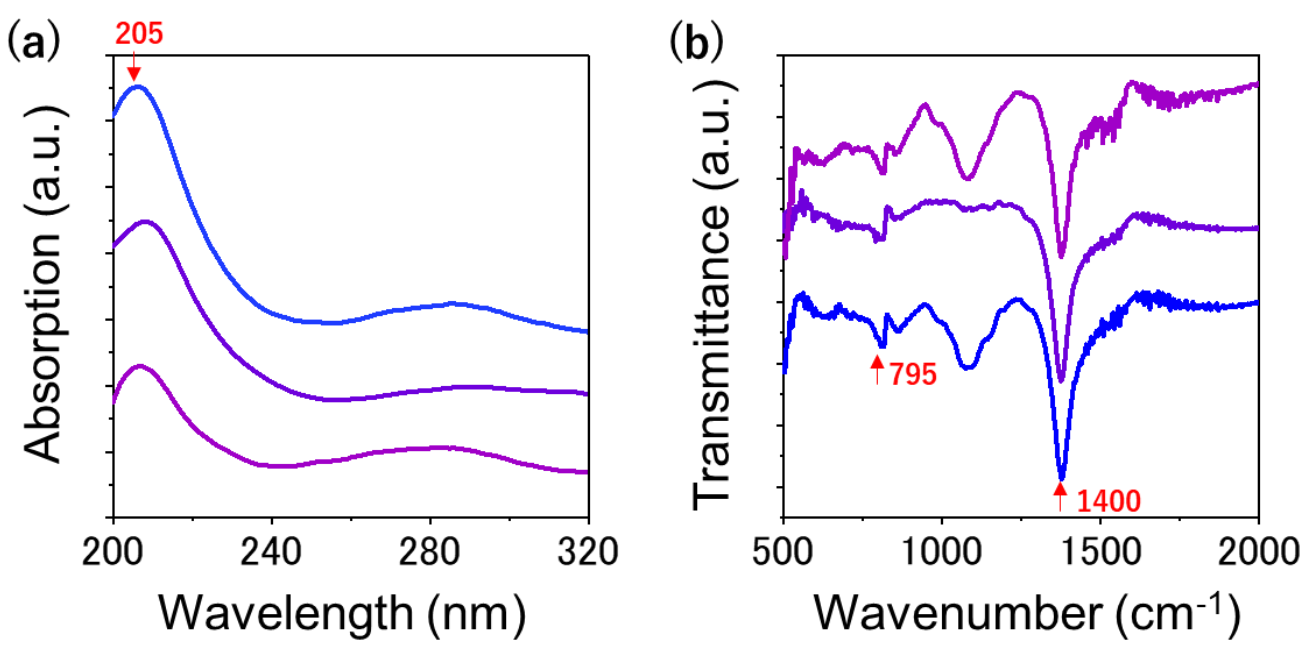

Figure S3. (a) UV-vis-NIR absorption and (b) Fourier transform infrared (FT-IR) transmittance spectra of the $\mathrm{hBN}$-grown CNT sheet grown with various conditions. 
(a)

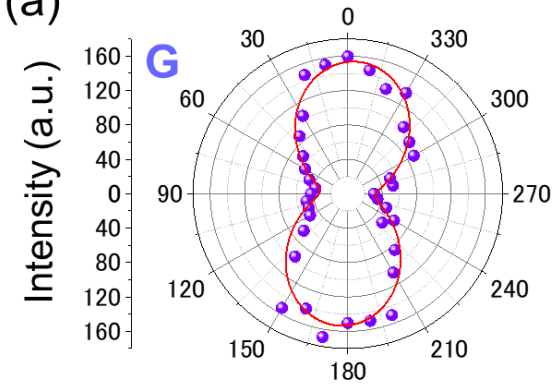

(c)

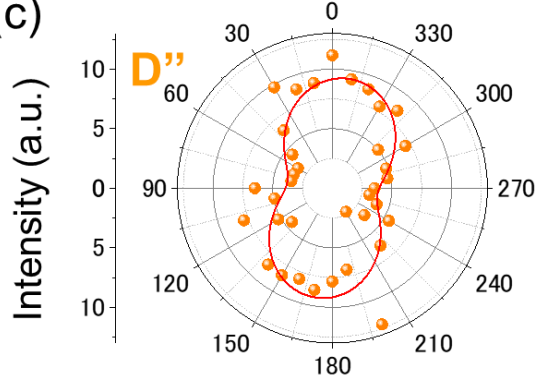

(b)

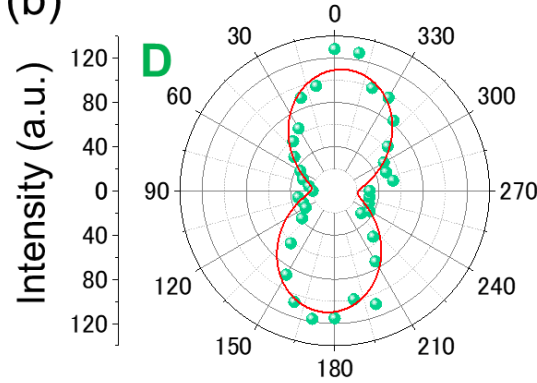

(d)

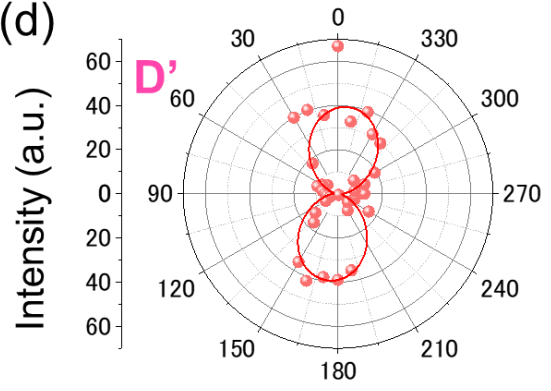

Figure S4. Polarization Raman measurement with pristine CNT sheet. Polar plots of the (a) G-, (b) D-, (c) D"-, (d) D'-band intensity obtained by peak fitting.
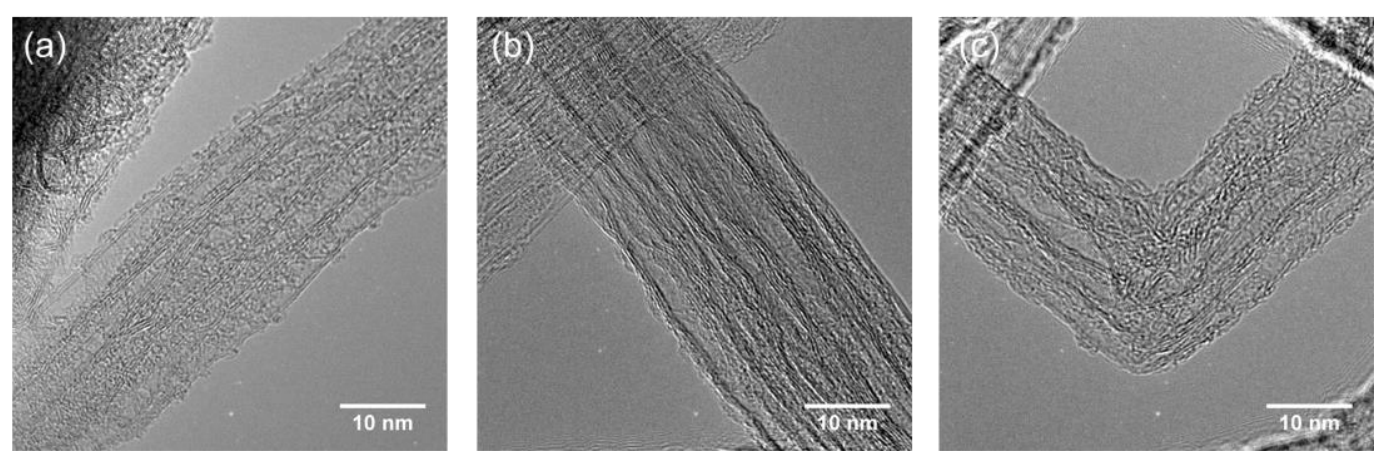

Figure S5. (a-c) TEM images of CNT bundles taken from internal of $\mathrm{hBN}$-grown CNT yarn. 


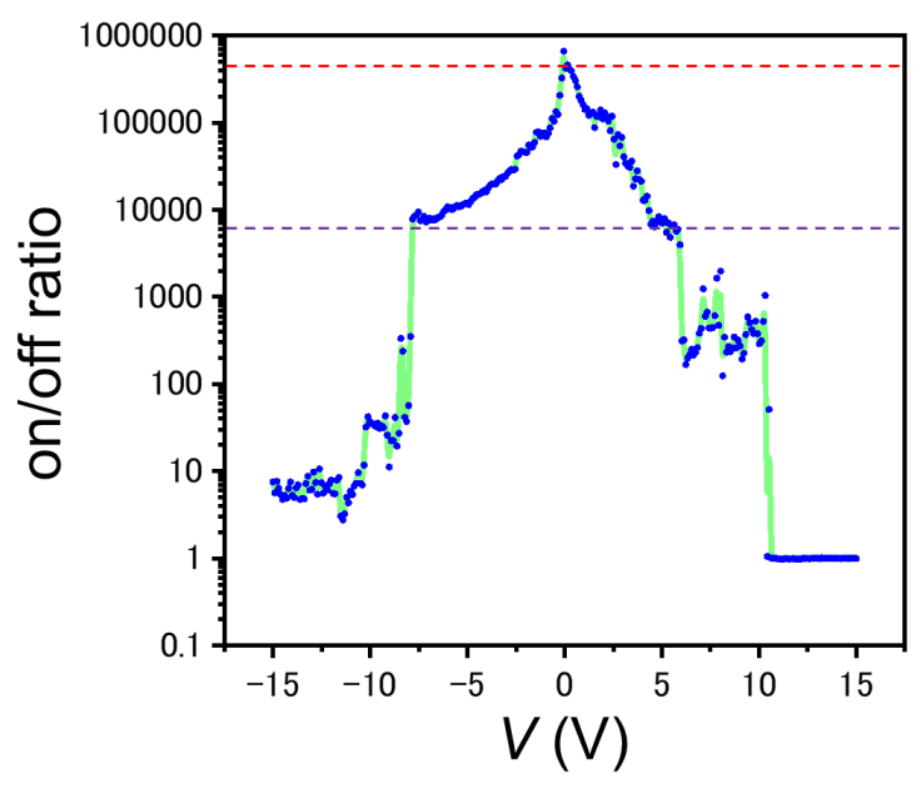

Figure S6. On/off ratio calculated with $I-V$ curve showing hysteresis.

(a)

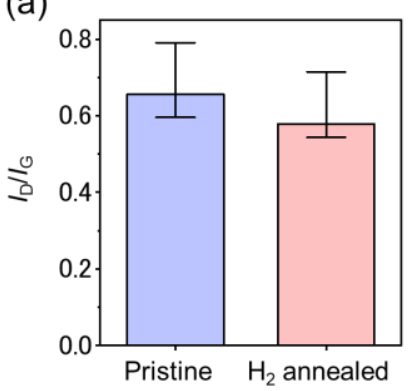

(b)

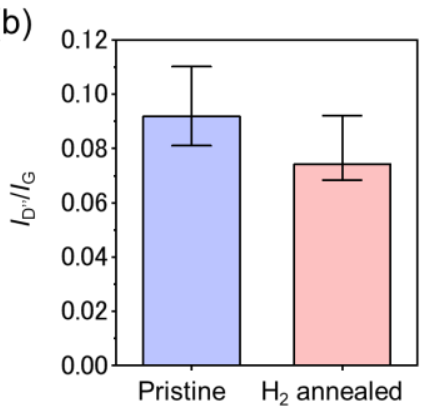

(c)

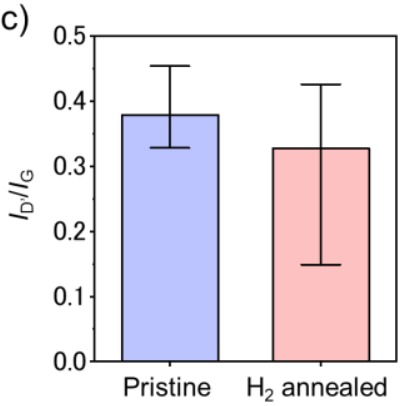

Figure S7. Normalized intensity of the (a) D-, (b) D' '-, and (c) D-band by the intensity of the G-band $\left(I_{\mathrm{D}} / I_{\mathrm{G}}, I_{\mathrm{D}}{ }^{\prime} / I_{\mathrm{G}}\right.$, and $\left.I_{\mathrm{D}}, I_{\mathrm{G}}\right)$ measured in pristine (light purple) and $\mathrm{H}_{2}$-annealed (light pink) CNT sheet. Ten points in each sample were corrected and averaged. 


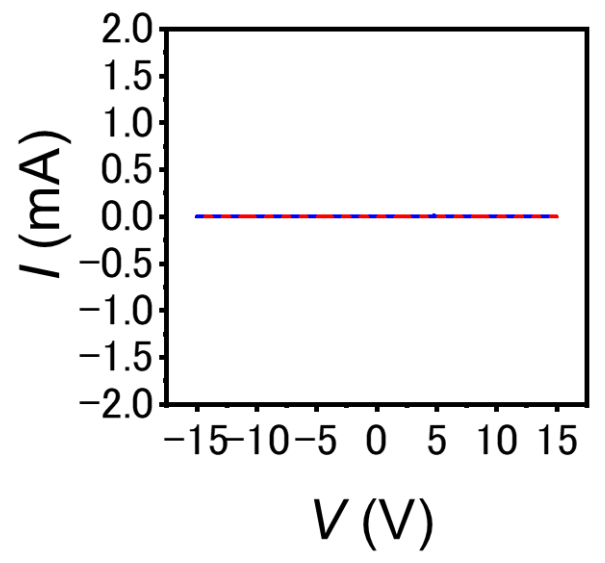

Figure S8. $I-V$ curves measured in hBN/CNT yarn prepared with the $\mathrm{hBN}$ growth condition of low $T_{\mathrm{g}}\left(1000{ }^{\circ} \mathrm{C}\right)$ and low $T_{\mathrm{s}}\left(90{ }^{\circ} \mathrm{C}\right)$ where we expect the orderly growth of the hBN layer on CNTs.

(a)

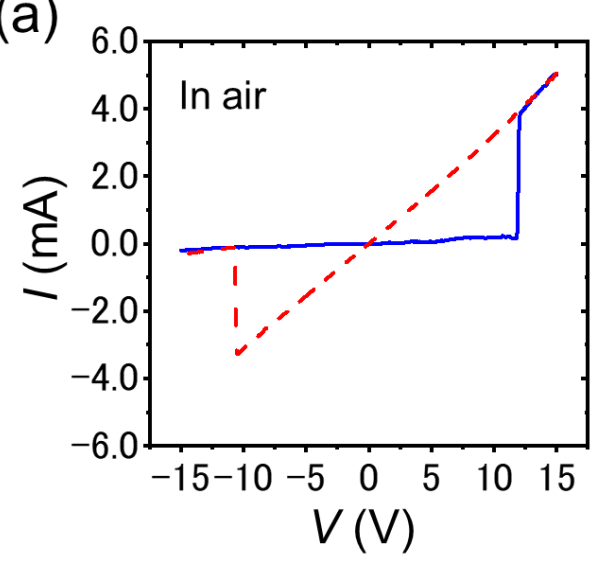

(b)

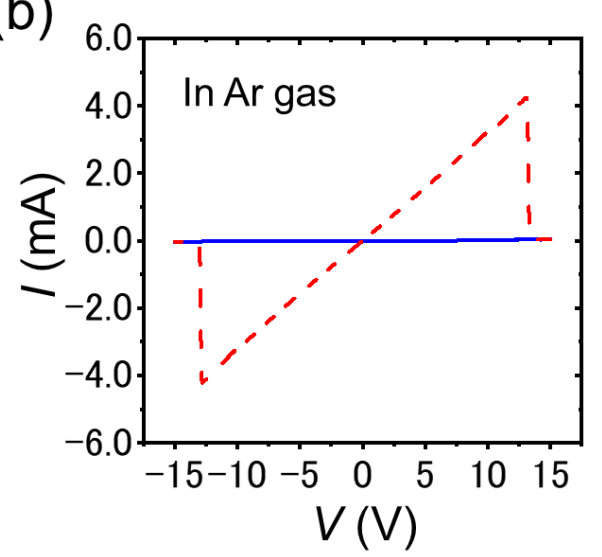

Figure S9. $I-V$ curves measured in (a) air and (b) Ar gas. 